
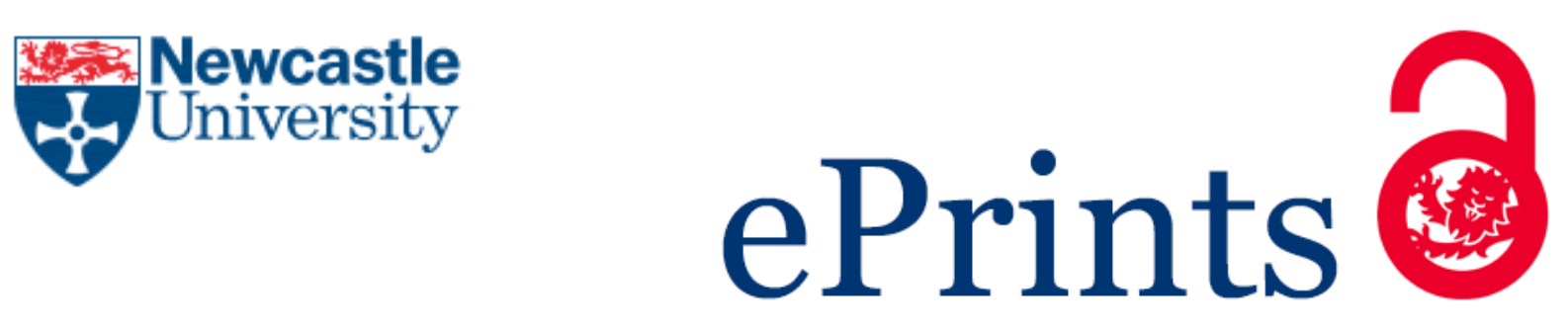

Srinivasaiah B, Tiwari R, Dhinagar S. Kalman Filter based estimation algorithm to improve the accuracy of automobile GPS navigation solution. In: SAE 2014 World Congress \& Exhibition. 2014, Detroit, Michigan, USA: SAE International.

\title{
Copyright:
}

Copyright (C) 2014 SAE International. This paper is posted on this site with permission from SAE International. It may not be shared, downloaded, transmitted in any manner, or stored on any additional repositories or retrieval system without prior written permission from SAE.

DOI link to article:

http://dx.doi.org/10.4271/2014-01-0268

Date deposited:

$12 / 03 / 2015$ 


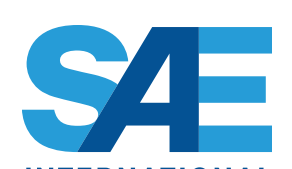

INTERNATIONAL

\section{Kalman Filter Based Estimation Algorithm to Improve the Accuracy of Automobile GPS Navigation Solution}

\section{Bhavani Srinivasaiah}

M/S TVS Motor Co., Ltd.

\section{Rajesh Tiwari}

Newcastle University

\section{Samraj Dhinagar}

M/S TVS Motor Co., Ltd.

CITATION: Srinivasaiah, B., Tiwari, R., and Dhinagar, S., "Kalman Filter Based Estimation Algorithm to Improve the Accuracy of Automobile GPS Navigation Solution," SAE Technical Paper 2014-01-0268, 2014, doi:10.4271/2014-01-0268.

Copyright @ 2014 SAE International

\begin{abstract}
The demand for location based services in automobile industry promoting applications in the area of telematics, vehicle to vehicle and infrastructure communications is encouraging research in the field of accurate navigation solutions. According to the $A B I$ research, position data is the prime enabler for above mentioned applications and the in-car navigation market growth is predicted to grow at $25.9 \%$ over the next five years. Consequently position, velocity and heading form the prime input state vector for the target applications. Global Positioning System is one of the accurate off-board sensors for navigation solution. Nevertheless, the cost and complexity of these systems are posing the biggest challenge to the automobile research engineers. Least squares estimation is one of the proven methods used for computing positioning solution under static conditions. However, its accuracy is considered to be poor in dynamic cases.
\end{abstract}

This work focuses on achieving accuracy within few meters for navigation solution using kinematic based position estimation algorithm. Real-time GPS raw data acquired by tracing a trajectory is further analyzed in a post-mission processing approach. Two positioning estimation algorithms are developed and tested for accuracy and precision. User trajectory obtained using recursive least squares resulted in poor accuracy and bias of about $10 \mathrm{~m}-15 \mathrm{~m}$. Subsequently, Kalman filter algorithm was developed to improve the overall accuracy of the kinematic system. The user trajectory for Kalman filter based algorithm was found to be smoother resulting in accuracy within $5 \mathrm{~m}$ with rare occasional outliers during GPS outages.

\section{Introduction}

In this electronic era, gadgets and electronic control systems have played a vital role in making human life comfortable. The phones are getting smarter and automobiles becoming more and more advanced. Furthermore the ever-increasing traffic congestion and road accidents urges for safer and communicative automobiles. Nevertheless, the cost and complexity of these systems are posing the biggest challenge to research and design engineers. A large number of researches are carried out in the field of vehicle navigation smart cars, automobile stability control and anti-collision systems [1,2, 3]; although haven't got any optimal design.

The science of vehicle navigation can be described in many ways even from ancient days. The starting point, arrival destination, direction of heading and vehicle speed are the main parameters required to commute from place to place efficiently. Hence, applications such as navigation, telematics and vehicle to vehicle communications require Position, Velocity and Attitude (PVA) as their primary input state vector. Global Positioning system (GPS) being one of the Global Navigation Satellite (GNSS) providers, can accurately determine positioning if its antenna has a clear visibility of sky. The GPS system comprises of a 32 satellite constellation and the receivers usually require the line of sight of atleast 4 satellites to compute positioning. However, the accuracy of the position computation algorithms employed is a big challenge for kinematic automobiles as against the implementation complexity and cost.

During the 1940s, Weiner designed a filter to estimate a specified random signal that is corrupted with noise so as to reduce the Mean square error of the random process. The filter uses a parametric estimation, in which the noise and signal priori is available with the system [4]. But this filter was 
designed only for continuous linear time-invariant systems. Later Kolmogorov demonstrated this filter for discrete-time systems. The linear time-variant version or the adaptive filtering technique of Wiener filter has been derived by Rudolf Kalman which is widely used for navigation applications [5].

The performance of the Extended Kalman Filter (EKF) for the navigation parameters integration was evaluated based on three main parameters: noise levels, sampling rate and curvature of the road in [6] by comparing the simulated data with Google Earth as reference. It was suggested that at higher curvature sampling rate to be kept higher to have bounded errors and a low sampling rate to get low Mean Square Error (MSE). Unscented Kalman Filter (UKF) proposed in [7] dealt with the EKF non-convergence during long GPS outages and involved complex state equations by eliminating the cascaded filters. This filter worked on non-linear state equation and had an advantage over other filters but with high cost of implementation. The Monte Carlo simulations and the field test revealed that the performance improvement of UKF was insufficient as against the EKF during GPS outages. Various versions of Kalman filter usage can be found in $[\underline{8}, \underline{9}, 10]$. The most widely suggested stochastic error model based on the vehicle dynamics trajectory was proposed in [11]. The sensor measurements formed the state vector and its performance was verified by performing the covariance analysis. The variations in the acceleration were used to correct the attitude estimate and the gyro bias which in turn improves the PVA measurements. The Covariance analysis performed in [5] using 3D or 2D position as its filter input for the observability analysis of error model was quite impressive. The simulations for different moving trajectories with an aiding filtering technique resulted in results that suffered from not considering real data.

The proposed Kalman filter based technique addresses some of the issues discussed in the literature. A good dynamic state error modeling combined with the most neglected real-time trajectory data analysis is considered for this work. This paper is organized into three main sections that describe the GPS with its signal structure and errors involved in measurements. It is then followed by the detailed description of the methodology implemented using least squares algorithm, error modeling for the Kalman filter and the trolley system used for tracing a trajectory to take the vehicle dynamics into consideration. The obtained results and findings were finally discussed briefly along with the conclusion.

\section{Global Positioning System}

The Global positioning system is one of the most popular providers for GNSS and include 3 segments for navigation system. The space segment comprises of the satellites and the control segment monitors the proper working of the satellites. The user segment is a gadget fitted in the automobile or handheld by the user as a device or smartphone. It consists of a GPS L1 antenna, RF amplifier and correlator encompassing the RF front-end along with the receiver and display units. The carrier frequency $L 1$ of the GPS signal lie in the $L$ - band range of $1-2 \mathrm{GHz}$ and is given by: fundamental frequency * $154=$
1575.42 MHz. L1 signal carries the C/A code (1.023 MHz) and navigation data $(50 \mathrm{~Hz})$ combined by the modulo- 2 adder and then imposed on the L1 carrier of $1575.42 \mathrm{MHz}$ as shown in figure 1 . The rate of change of phase is expressed in terms of chipping rate of $1.023 \mathrm{Mbps}$ with phase variation between 0 and $\pi$. To obtain the pseudorange, 3 subframes are adequate and take about 18 seconds. To compute the user position 3 subframes from atleast 4 satellites are required. The other 2 subframes provide the support data. Unfortunately, all the 3 subframes from the 4 satellites don't reach the receiver at the same time and the receiver also doesn't know the starting point of the subframes. Hence, around 30-40 seconds of data is required to estimate the initial user position [12].

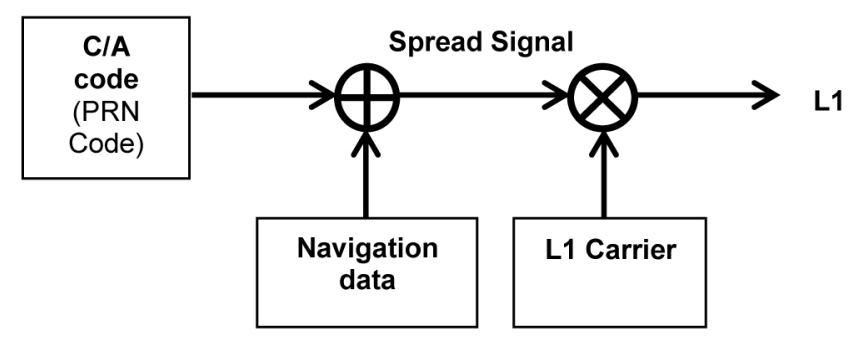

Figure 1. L1 signal generation

\section{GPS Signal Sructure}

Mathematically, the L1 signal broadcasted by the GPS satellites is given by $S_{L 1}^{p}(t)$ :

$$
\begin{aligned}
& S_{L 1}^{p}(t)= \\
& A_{p} P^{p}(t) D^{p}(t) \cos \left(2 \pi f_{1} t+\varphi\right)+A_{c} G^{p}(t) D^{p}(t) \sin \left(2 \pi f_{1} t+\varphi\right)
\end{aligned}
$$

where, $\mathrm{f}_{1}=\mathrm{L} 1$ carrier frequency, $P^{p}(t)=$ phase of $\mathrm{P}(\mathrm{Y})$-code, $D^{p}(t)=$ Navigation data,

$A_{p} \& A_{c}$ are the amplitude of $\mathrm{P}(\mathrm{Y})$ code and $\mathrm{C} / \mathrm{A}$ code respectively, and

$G^{p}(t)=$ phase of C/A-code and $\varphi=$ initial phase.

\section{GPS Errors}

The accuracy of the estimated user position by the receiver depends on many factors along with the satellite geometry. The user position estimation depends on the pseudorange measurements which can be affected by parameters such as multipath fading, ionosphere and tropospheric delays, receiver clock errors and etc. Some of the major ranging error sources that require mitigation techniques are described in $[13, \underline{14}, \underline{15}]$ and discussed briefly in this section.

The atomic clock within the satellites is very accurate and its information is carried by the GPS signals. Using this information, receiver clocks are synchronized with atomic clock during the positioning. Since the receiver clocks are not the accurate ones, clock anomalies are always present and account for few meters of inaccuracy in the estimation. 
The positioning solution is good if more than 4 satellites are available to the receiver but can be more accurate if the satellites satisfy the principle of satellite geometry and the method of finding the best geometry for receiver is termed as Dilution of Precision (DOP).

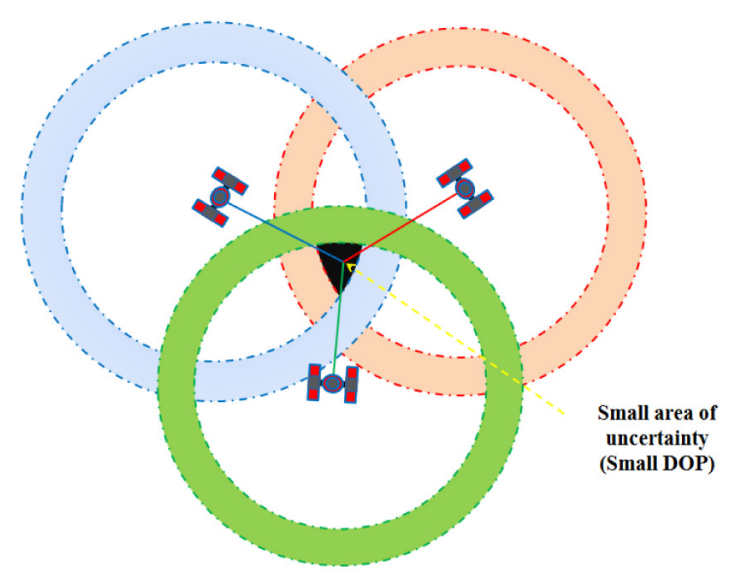

(a)

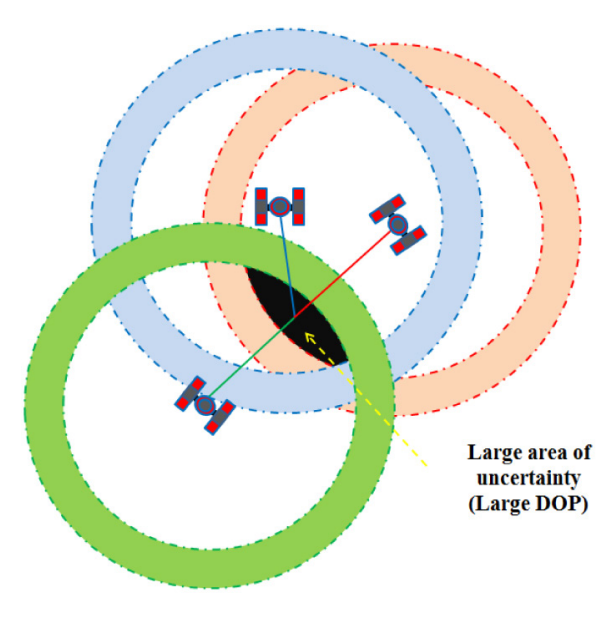

(b)

Figure 2. (a) Good satellite Geometry and (b) Bad satellite Geometry modified from [12]

Figure 2 illustrates the area of convergence for two cases: in the first case, the smaller area of uncertainty enables the easy computation of user positioning; whilst in the second case, the area of uncertainty is large that makes the receiver decision difficult. This is because of the tangential surface of the satellite range measurements that gives more data points far apart from each other. This ultimately leads to substantial range measurement errors. The troposphere/ionosphere delays and multipath are other sources of error that can be addressed for further improvement in position computation.

\section{Methodology}

The prime motivation behind this work is to develop a navigation algorithm that not only performs reliably at diverse GPS signal outages situation but also feasible and costeffective for the automobile market. A flexible and effective estimation algorithm based on Kalman filter is developed for GPS receiver. The algorithm developed was tested for outdoor performance using a trolley system.

\section{Block Diagram}

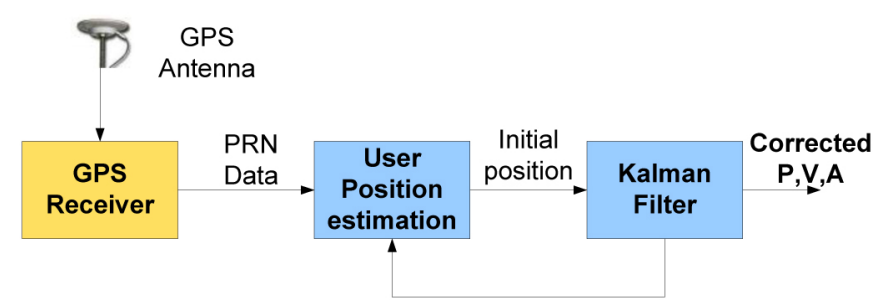

Figure 3. Block diagram of the GPS navigation solution computation

The overall system used for developing and testing the algorithm is illustrated in figure 3 . The satellite broadcast signals are received by the GPS antenna that is a part of the RF front-end. The raw GPS data is captured and stored on the SD card by the receiver. These navigation and observation files were further converted to user readable format using file conversion software for further processing. The GPS receiver estimates the positioning based on the locked satellite data received by the GPS front-end. In Kalman filter, the covariance matrix and the state vector gets updated every epoch during normal conditions and corrected during GPS outages based on the parametric model designed and previous input state vector. Since Kalman filter works on the prediction of state vector using previous inputs, the initial estimate for the Kalman filter is provided by the least squares estimation of positioning.

\section{User Positioning using Least Squares}

The PRNs locked by the receiver aid in estimating the range between satellite-receiver link and satellite position from navigation data present within the received signal. These parameters are further used in computing position using recursive Least Squares Estimation technique. The three user coordinates and the clock bias error accounting for four unknowns were solved. Hence, good results were achieved by taking all the available satellites into consideration for calculating the user position.

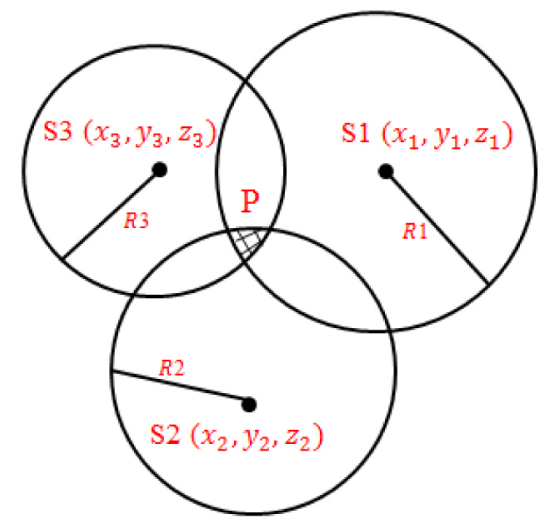

Figure 4. Trilateration of 3 satellites

As seen from figure 4, the user position is calculated from the pseudorange $R_{i}$ i.e., the distance between satellite and user, where $i=1,2, \ldots \ldots \ldots n ; n=$ number of satellites. 


$$
\frac{R_{i}=}{\sqrt{\left(x_{u}-x_{i}\right)^{2}+\left(y_{u}-y_{i}\right)^{2}+\left(z_{u}-z_{i}\right)^{2}}+b_{u}}
$$

$\mathrm{P}$ is the user at $\left(x_{u}, y_{u}, z_{u}\right) \& b_{u}$ is the user clock bias; $\mathrm{S} 1, \mathrm{~S} 2$ and $\mathrm{S} 3$ are the 3 satellites.

Hence, by adopting recursive Least-squares method the following equation satisfies the finding of the solution.

$$
\vec{X}=H \vec{\theta}+\vec{\omega}
$$

$$
\vec{X}=\left[\begin{array}{l}
\hat{R}_{1}-R_{n 1} \\
\hat{R}_{2}-R_{n 2} \\
\hat{R}_{3}-R_{n 3} \\
\hat{R}_{i}-R_{n i}
\end{array}\right], H=\left[\begin{array}{lll}
\frac{\left(x_{n}-x_{1}\right)}{R_{n 1}} & \frac{\left(y_{n}-y_{1}\right)}{R_{n 1}} & \frac{\left(z_{n}-z_{1}\right)}{R_{n 1}} \\
\frac{\left(x_{n}-x_{2}\right)}{R_{n 2}} & \frac{\left(y_{n}-y_{2}\right)}{R_{n 2}} & \frac{\left(z_{n}-z_{1}\right)}{R_{n 2}} \\
\frac{\left(x_{n}-x_{3}\right)}{R_{n 3}} & \frac{\left(y_{n}-y_{3}\right)}{R_{n 3}} & \frac{\left(z_{n}-z_{1}\right)}{R_{n 3}} \\
\frac{\left(x_{n}-x_{i}\right)}{R_{n i}} & \frac{\left(y_{n}-y_{i}\right)}{R_{n i}} & \frac{\left(z_{n}-z_{i}\right)}{R_{n i}}
\end{array}\right],
$$$$
\vec{\theta}=\left[\begin{array}{l}
\Delta x \\
\Delta y \\
\Delta z
\end{array}\right] \& \vec{\omega} \quad \text { is the noise vector. }
$$

The updated change in position is given by,

$$
\vec{\theta}=\left(H^{T} C^{-1} H\right)^{-1} H^{T} C^{-1} \vec{X}
$$

The new position is calculated using

$$
\begin{aligned}
& x_{\text {new }}=x_{\text {old }}+\Delta x ; \quad y_{\text {new }}=y_{\text {old }}+\Delta y ; \\
& z_{\text {new }}=z_{\text {old }}+\Delta z ; \quad b_{\text {unew }}=b_{\text {uold }}+\Delta b_{u}
\end{aligned}
$$

where $\Delta x, \Delta y, \Delta z$ are the errors in measurements.

The iteration is repeated until a pre-assigned threshold is reached.

$$
\left(\Delta x_{k}\right)^{2}+\left(\Delta y_{k}\right)^{2}+\left(\Delta z_{k}\right)^{2} \leq \text { threshold }
$$

The sensitivity matrix $(H)$ is used for the calculation of DOP.

$$
\left(H^{T} H\right)^{-1}=\left[\begin{array}{llll}
D_{11} & D_{12} & D_{13} & D_{14} \\
D_{21} & D_{22} & D_{23} & D_{24} \\
D_{31} & D_{32} & D_{33} & D_{34} \\
D_{41} & D_{42} & D_{43} & D_{44}
\end{array}\right]
$$

Geometric DOP (GDOP) is the most common parameter related to DOP that indicates the accuracy of errors due to satellite geometry.

$$
G D O P=\sqrt{D_{11}+D_{22}+D_{33}+D_{44}}
$$

The GDOP have to be as low as possible to have a better result for positioning. The GDOP confidence levels between the range 1 to 2 is considered the best choice of satellite positions, and between 2 to 5 is also considered favorable for use in land navigation [13].

\section{Kalman Filter Error Model}

Kalman filter is a technique that works excellently for the time variant systems with uncertain dynamics in estimating the state vector by combining noisy sensor measurements/outputs. It is a parametric estimation method where in the state vector parameters are estimated based on the priori. Kalman gain used to control the covariance and a new estimated update is provided for the state vector. In case of huge drifts and signal loss like GPS outages for few seconds, a corrected estimate is provided for the next loop of state vector.

i. The state dynamic model that involves the observables also termed as state vector and the dynamics of the vehicle affecting the observables. This also includes the state transition matrix and the process noise covariance matrix.

ii. The measurement model containing the measurement vector, measurement sensitivity matrix and the measurement noise covariance matrix [13].

iii. Numerous methods are possible by choosing a good combination of the state variables and that will decide the level of integration. Careful selection of state variables affects the performance and the precision of the integrated system.

GPS positioning along with velocity and attitude information can be updated continuously using the Kalman filter algorithm. GPS data was logged at $10 \mathrm{~Hz}$. Although the initial values were taken by least squares method, Kalman filter takes over for the recursive estimation and updating. The positioning is updated every epoch at good GPS conditions and predicted in case of GPS outages.

Kalman Filter utilizes the priori information at the system and predicts the next measurement. It estimates the uncertainty of its prediction by comparing the priori/predicted values with the measurements. It is a recursive system that works on the principle of weighed averaging on a range of noisy measurement to provide a precise prediction on the future and measured values. 


$$
\begin{gathered}
\text { Input equation } \longrightarrow \quad x_{i+1}=\underbrace{A_{i} x_{i}+B_{i} u_{i}}_{\text {Observables }}+n_{s i} \\
\text { Output equation } \longrightarrow y_{i}=E_{i} x_{i}+n_{m i} \\
\searrow \\
\text { System uncertainty } \\
\text { Measurement uncertainty }
\end{gathered}
$$

The equations (9) represent the input and output equations for any noisy sensor measurements and it clearly exhibits that the system can be viewed as sum of a deterministic system and a stochastic system. Since we know the behavior of the deterministic system by making them as observables; let us further investigate the stochastic system which is nothing but we call the error modeling. $x_{i}$ represents the system state vector that cannot be measured. $y_{i}$ the output of the system state vector that is measured by the sensor. $E_{i}$ is the transition matrix that can be used to estimate the input state vector. The uncertainty in the input is termed as the system or the process noise vector whereas the uncertainty in the output is termed as the measurement noise vector. The same principle is employed in the design of Kalman filter along with the following assumptions [13].

a. The system is linear and dynamic.

b. The process and measurement errors are Gaussian distributed with zero mean and a known variance. Since it's a zero mean process the correlation is the same as covariance.

c. Process noise and measurement noise are statistically uncorrelated.

Practically, most of the problems encountered in navigation solutions are non-linear considering the non-linearity in the dynamics of the vehicle. The working principle is as shown in figure 5 along with the mathematical expressions involved in Kalman filter are as shown in table 1.

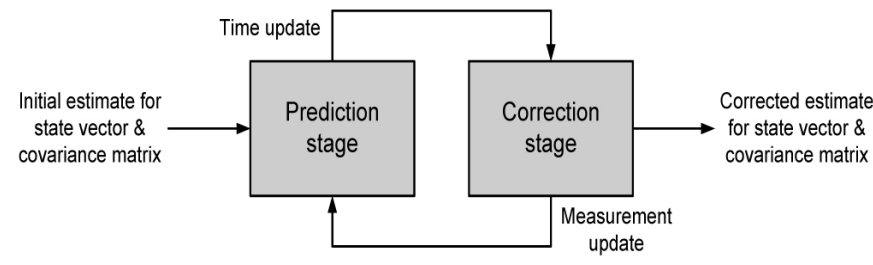

Figure 5. Block diagram to illustrate the working of Kalman filter
Table 1. Kalman Filter equations [28]

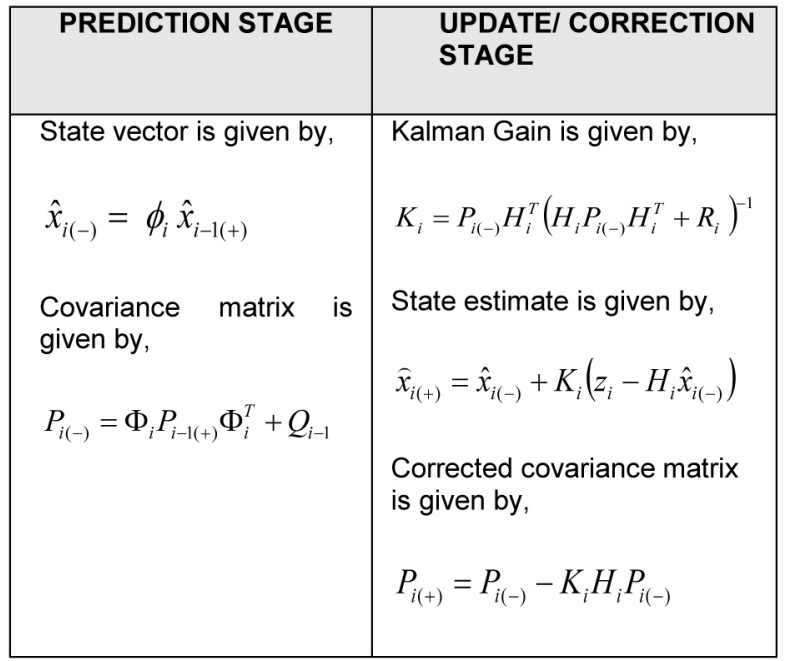

$P_{i(-)}$ and $\hat{x}_{i(-)}$ are the predicted/ initial estimate of covariance matrix and state vector, where $i$ represents the time index. $H_{i}$ represents the measurement sensitivity matrix and $K_{i}$ is the Kalman gain. $\Phi_{i}$ is the state transition matrix, $Q_{i-1}$ represents the covariance of noise uncertainties and $R_{i}$ is the measurement uncertainties. $P_{i(+)}$ and $\widehat{x}_{i(+)}$ are the corrected estimate of covariance and state vector.

\section{Investigation of Positioning Solution with Kinematic Behavior}

The kinematic conditions were further investigated using a trolley fitted with the GPS sensor along with its GPS front-end embedded in the receiver and the GPS antenna as shown in figure 6.

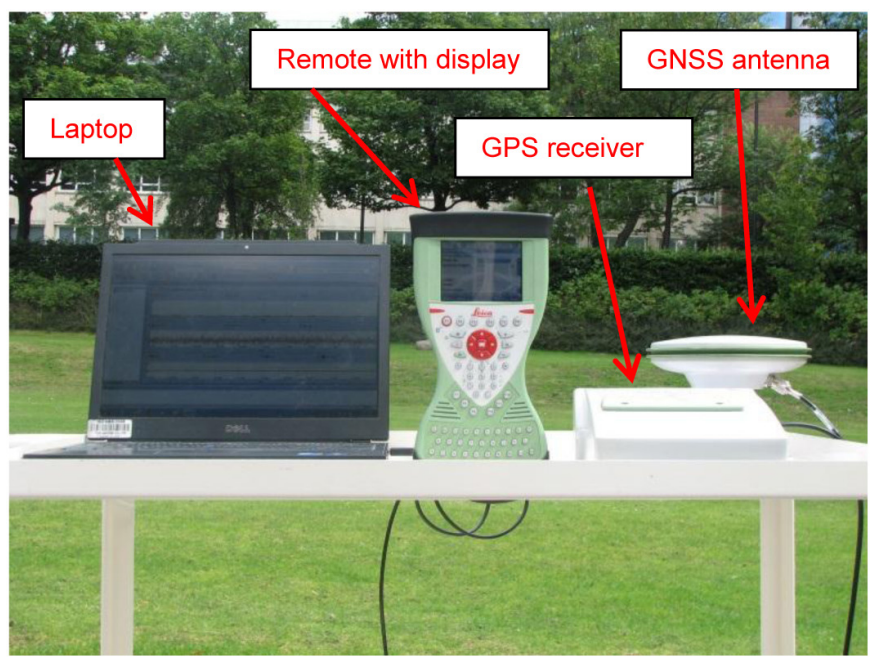

Figure 6. Trolley system test setup

A trajectory was traced within the Newcastle university campus, United Kingdom using the trolley and raw data was acquired concurrently for the post processing and analysis. The trajectory traced is as shown in figure 7. 


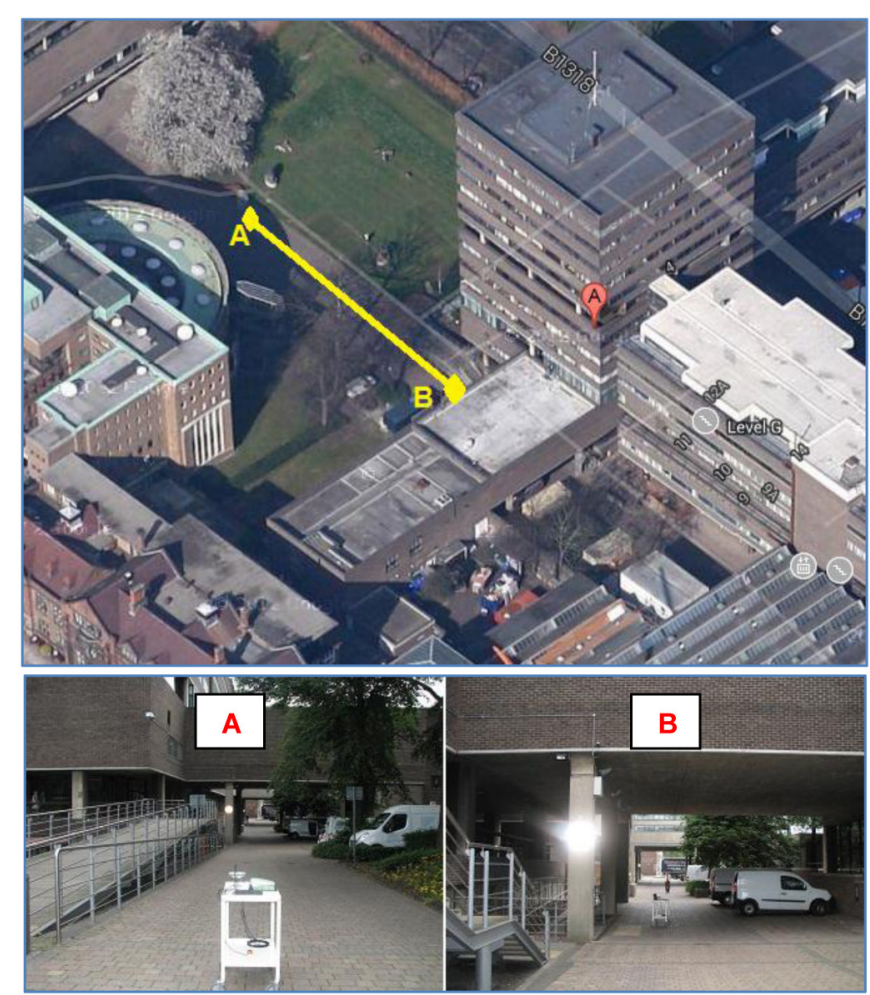

Figure 7. Trajectory trace on Google map

The trajectory surveyed using Google maps shown in figure 7 was chosen carefully so that it initially at point A with open area gets probably good satellite visibility for few minutes. Then the trolley was moved into the tunnel of the buildings for about $5 \mathrm{~s}$ as shown with point $B$, so that the satellite lock drops to zero or one with probable multipath. Then the trolley was returned to the point $A$ for completing the trace. This experimentation was repeated during morning and evening on the same day to observe the satellite positions which displayed a significant change in satellite visibility and geometry throughout the day. Furthermore, more than 7 satellites were locked whereas about 4 satellites were locked during the morning. The whole experiment was carried out for about 15 minutes with a halt at points $A$ and $B$ for about 5 and 2 minutes respectively. Moreover, same experiment was repeated for 2 more days for data acquisition with different trials.

\section{Results and Discussion}

The results obtained initially for GPS acquired signal was investigated for their data acquisition eminence and the static behavior. Furthermore, kinematic tests were conducted using a trolley for real time data acquisition under different GPS conditions. Eventually, GPS system with least squares estimation and an efficient system using Kalman filter were developed, tested and analyzed for their performance. The MATLAB algorithms developed were used for validation of the system for the described trolley trajectory in a post-mission processing approach. The GPS satellite signals at a particular epoch received by the antenna were fed into the front-end to obtain the locked satellite IDs.

\section{Acquisition of Satellites}

The developed algorithm was used for validation of the system for the described trolley trajectory in a post-mission processing approach. The GPS satellite signals at a particular epoch received by the antenna were fed into the front-end of the receiver to obtain the locked satellite IDs. Figure 8 gives the result summary of the satellite acquisition. The satellite acquisition was successful as shown in figure 8(a) that is good enough to calculate the user positioning. Figure 8(a) gives the power spectral density of the received signal after despreading and centered at the intermediate frequency set to 6.5 $\mathrm{MHz}$ in the receiver. Figure 9(a) and (b) represents the PRNs locked and not locked by the receiver respectively. A locked PRN is represented by a single power peak at the intermediate frequency and its corresponding code phase.

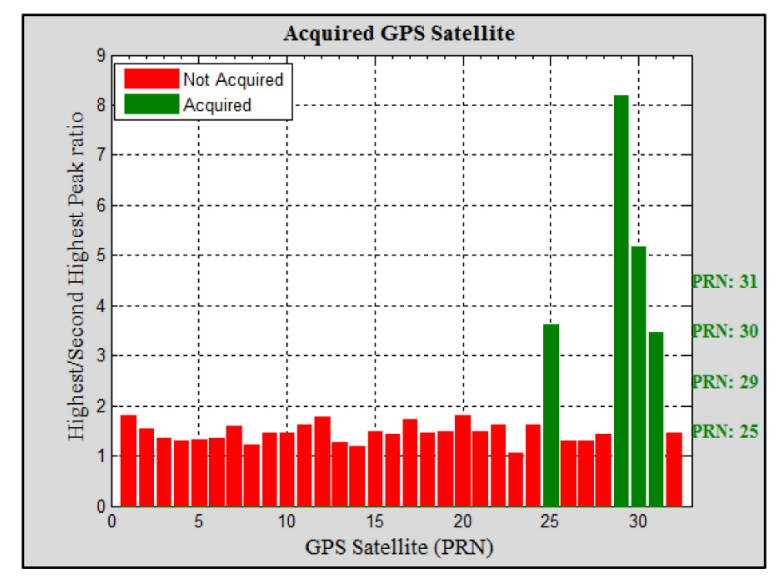

(a)

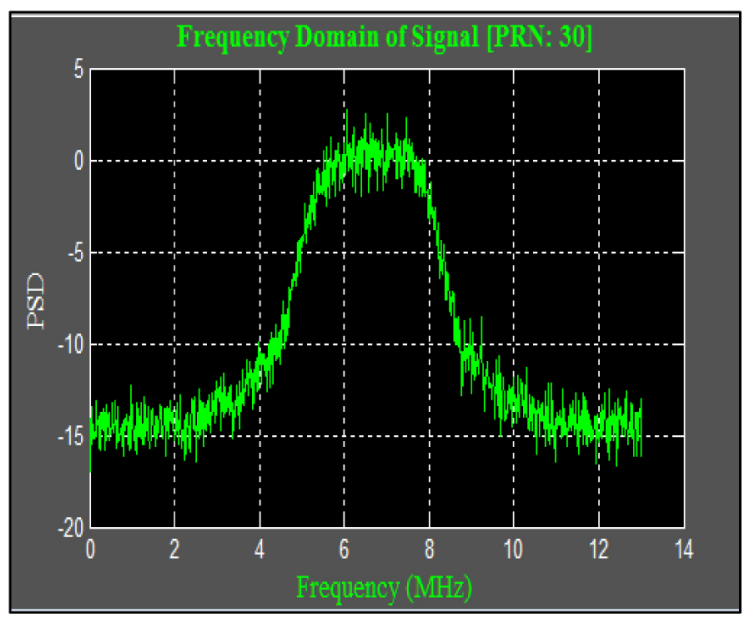

(b)

Figure 8. GPS signal Acquisition-(a) Number of satellites locked, (b) Received signal in Frequency Domain 


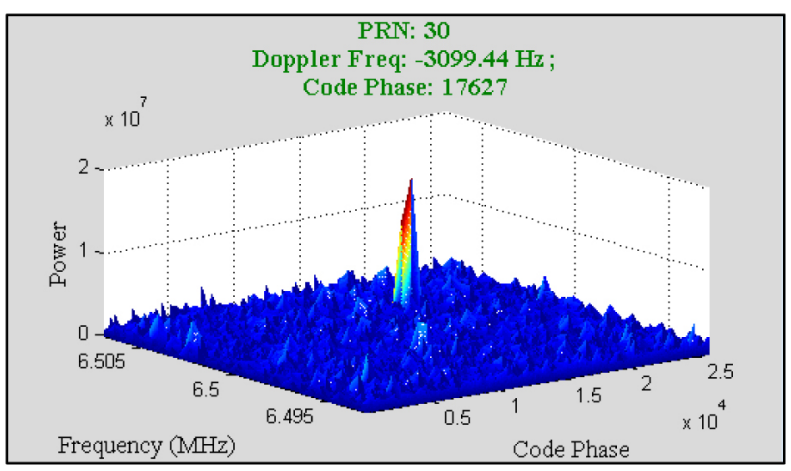

(a)

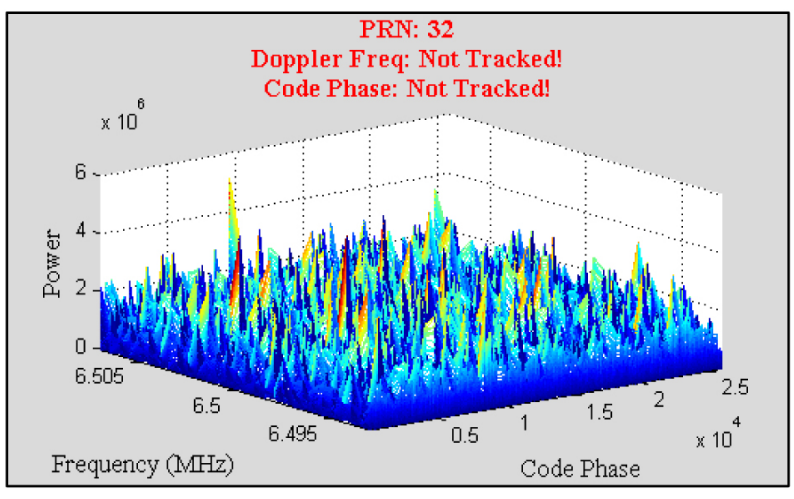

(b)

Figure 9. (a) PRN locked \& (b) PRN not locked

\section{Position Solution using Recursive Least Squares}

The random noise effect due to the motion is a threat which could be illustrated in the achieved results. Due to the dynamic noise, the accuracy levels were low in meters as compared to the stationary reciver positioning. The idea behind studying the behaviour of this noise along with satellite geometry was to repeat the same experiment at the same time on different days. This also justified that the same PRNs happen to position in the same place twice a day so that we were able to obtain a constant pattern of the noise. Two tests were conducted using trolley during different time of the day.

The same test was repeated in the evening and with an excellent GDOP of about 2.4 with 8 satellites locked at point A. GPS positioning was computed using the recursive least squares and the results was quite satisfactory as shown in figure 10 . The positioning convergence with a similar pattern of the actual trajectory trace was observed with a positional bias of about $7 \mathrm{~m}$. The accuracy level achieved is comparable with the commercially available GPS receiver that is about $15 \mathrm{~m}$ without the augmentation system support. Both the tests conducted above at different time with good and bad satellite geometry revealed that recursive least squares estimation works excellently for static GPS receivers. For automobile applications with dynamic motion patterns least squares does not provide accurate results. Fortunately, the accuracy could be improved with further processing the raw data and corrections for clocks along with an estimation algorithm for time-variant systems.

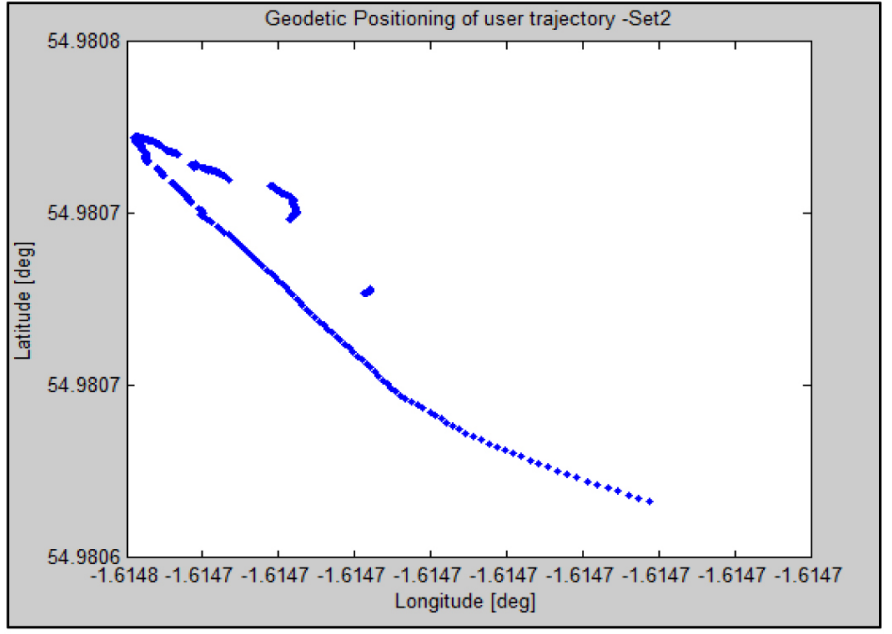

(a)

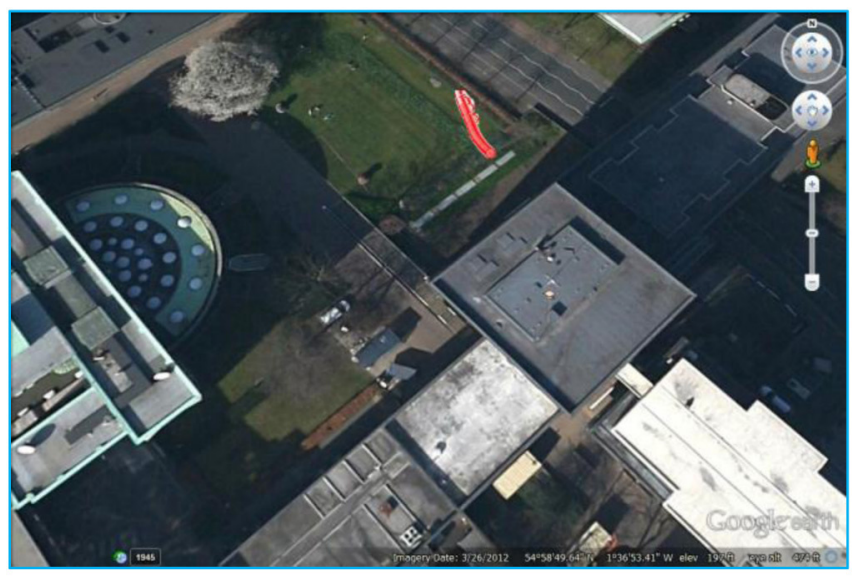

(b)

Figure 10. Trajectory for Positioning - (a) geodetic plot, (b) Google Earth plot

\section{GPS Position Solution using Kalman Filter}

Kalman filter algorithm was implemented for further processing of the first position obtained by least squares to improve the overall accuracy. It predicts and corrects the next position based on the error model matrix designed and the previous position sample provided by the least squares method by further updating the covariance matrix.

The Kalman filter output was found to be smoother as compared to the least squares as shown in figure 11. But the initial value convergence was not compliant to the original position since the Kalman filter takes some iteration to correct the solution. It was also noted that the GDOP was about 5.3 for the data and availability of 4 satellites resulted in low accuracy of the result. Furthermore the noise due to the dynamics of the trolley and the surrounding building also contributed towards error. In addition, the hardware GPS receiver provided the pseudorange that accumulates for the error in position solution. Hence clock errors were addressed for the accuracy improvement. 


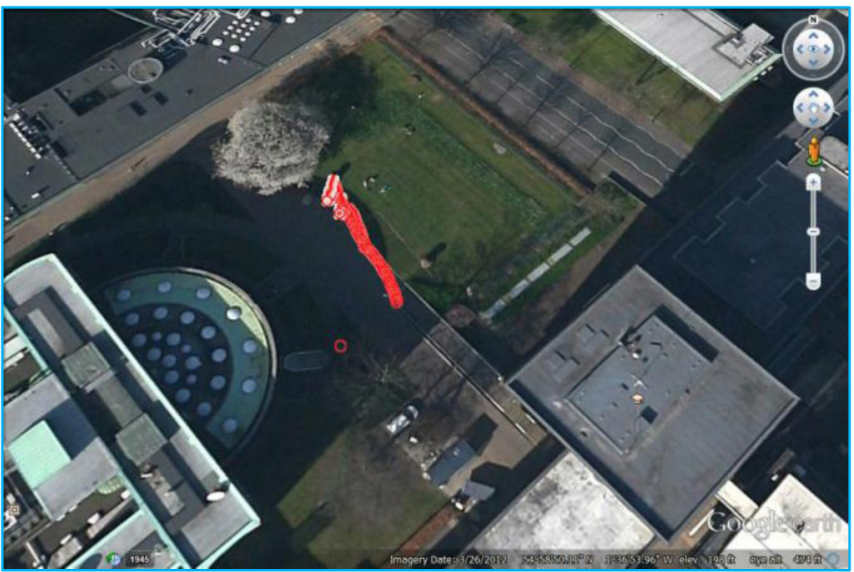

Figure 11. Trajectory for user positioning using Kalman filter

The orbital errors were also corrected and the data set was obtained for estimation. A smooth trajectory trace was obtained with accuracy of $2 \mathrm{~m}$ as shown in figure 12 . As demonstrated earlier the Kalman filter takes some iteration to converge to the actual position. Hence some of the initial few position estimates can be neglected.

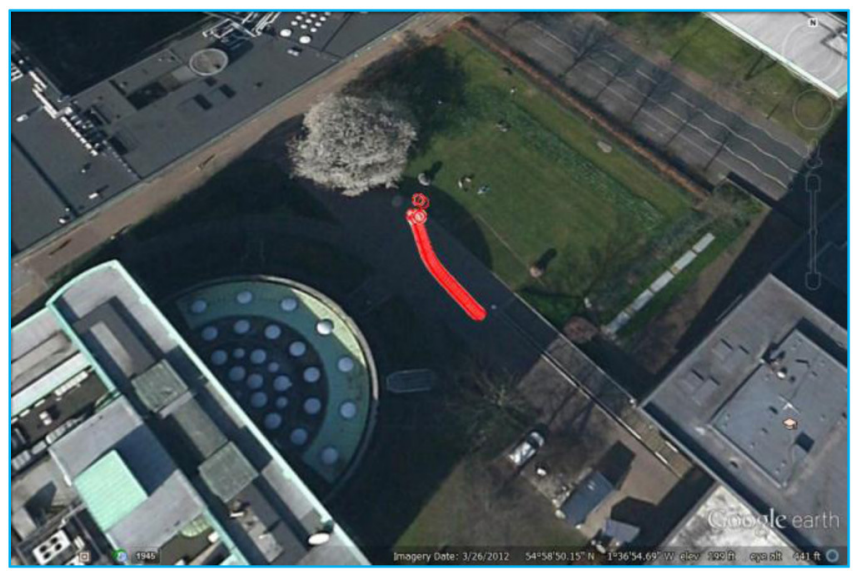

Figure 12. Trajectory for user positioning using Kalman filter

\section{Conclusions}

The contribution of this work was to develop algorithms for the GPS receiver to improve the accuracy of the navigation solution for automobile applications. Two signal processing methods were developed and investigated. The problems related to the GPS pseudorange errors, satellite and receiver clock errors were dealt with. Real time data collection with all the captured dynamic behavior and noise experiment was repeated to acquire GPS raw data at different times of the day to study the error pattern occurred due to the satellite geometry. Subsequently raw data was acquired for more days during the same time to investigate the dynamic noise statistical characteristics. The main algorithm developed uses a Least squares sub-program for computing the first position. Later the flow is taken over by another sub-program based on Kalman filter for predicting and updating the dynamic behavior of the trolley to estimate position solution. The following points outline the conclusions drawn from the experimentation and analysis of test results: i. With clear sky providing the continuous update of the satellite signal and a bad satellite geometry of only 4 satellites resulted in very low accurate position solution for the least squares algorithm. For stationary time of 1 minute the accuracy was about $5 \mathrm{~m}$ but the solution did not converge when the trolley was being set to motion.

ii. For the same algorithm but with good satellite geometry of 2.4 GDOP and 8 satellites locked, least squares algorithm gave same trajectory pattern but with a bias in the positioning of about $7 \mathrm{~m}$. The achieved results revealed that a more sophisticated algorithm was required to deal with the dynamic aspects.

iii. Kalman filter algorithm for position estimation gave more accurate results with accuracy of around $2 \mathrm{~m}$ apart from the few initial deviations taken during the correcting and convergence process.

iv. The satellite clock errors and the receiver clock bias along with the orbital errors correction model was incorporated in the algorithm and achieved smoother trajectory with position solution accuracy within $2 \mathrm{~m}$ for the dynamic behavior.

The developed algorithm works well with the GPS outages for few seconds. Based on the results and findings of this work, following recommendations are proposed that would be interesting to continue further with the research. Eventually, aided systems work well especially low cost MEMS inertial sensors, vision based system and etc. that promotes the navigation system to sustain longer GPS outages. The integration techniques are fairly complex in terms of selecting the observables and the state vector formation which are in-turn dependent strongly on the criticality of the applications. Additionally, they play a vital role in directing the level of integration as well as the accuracy and reliability of the overall system.

\section{References}

1. SAE International, "Designers using GPS data for powertrains, apps, other systems," http://www.sae.org/ mags/aei/elect/12060, accessed on 21.08.2013.

2. ChangP. and Lin J. M., Lo Y., "Integrated Smart Car Navigation and Voice Control System Design", 32nd Annual Conference IEEE Industrial Electronics, IECON 2006.

3. Sato Y., Shimonaka Y., Maruoka T., Wada T., Okada H., "Vehicular Collision Avoidance Support System v2 (VCASSv2) by GPS+INS Hybrid Vehicular Positioning Method", Australasian Telecommunication Networks and Applications Conference, December 2007.

4. Kalman Filter, http://en.wikipedia.org/wiki/Kalman Filter, Accessed on 18.04.2013.

5. Hayes M.H., "Statistical Digital Signal Processing and Modeling, John Wiley \& Sons”, INC, 1996.

6. Kim S. B., Bazin J. C., Lee H. K., Choi K. H., Park S. Y., "Ground vehicle navigation in harsh urban conditions by integrating inertial navigation system, global positioning system, odometer and vision data", IET Radar Sonar Navigation, Vol. 5, Iss. 8, pp. 814-823, 2011. 
7. Navigation with more than GPS, Navigation applications, http://autoelectronics.com/telematics/navigation_systems/ navigating-with-more-than-just-gps-0517I, accessed 06.03.2013.

8. Yu J., Chen X., "Application of Extended Kalman Filter in Ultra-Tight GPS/INS Integration Based on GPS Software Receiver", International Conference on Green Circuits and Systems (ICGCS), 2010.

9. Karatsinides S. P., "Enhancing Filter Robustness in Cascaded CPS-INS Integrations", IEEE Transactions on Aerospace and Electronic Systems, Vol. 30, No. 4 October 1994.

10. Ojha U., Chow M., Chang T. and Daniel J., "Analysis on the Kalman Filter Performance in GPS/INS Integration at Different Noise Levels, Sampling Periods and Curvatures", 35th Annual Conference of IEEE, Industrial Electronics, IECON 2009.

11. Dutt S.I., Rao G.S. B., Rani S.S., Babu S. R., etc., "Investigation of GDOP for Precise user Position Computation with all Satellites in view and Optimum four Satellite Configurations", J. Ind. Geophys. Union, July 2009, Vol.13, No.3, pp.139-148.

12. Tsui J.B., "Fundamentals of global positioning system receivers: A software approach", John Wiley \& Sons Inc., 2nd edition, 2005.

13. Grewal M.S., Weill L.R. and Andrews A.P., "Global Positioning Systems, Inertial Navigation, and Integration", Second Edition, Wiley, 2007.

14. Sharawi M. S., Korniyenko O. V., "Software Defined Radios: A Software GPS Receiver Example", IEEE/ACS International Conference on Computer Systems and Applications, 2007.

15. FarrelJ., Barth M., "The global positioning system and inertial navigation”, McGraw-Hill publications, 1999.

\section{Contact Information}

Bhavani Srinivasaiah
Member, R\&D
TVS Motor Company Ltd.
PB No.4, Harita
Hosur - 635109
TamilNadu, INDIA
s.bhavani@tvsmotor.co.in
http://tvsmotor.in
Dr. Rajesh Tiwari
School of Electrical and Electronic Engineering
Merz Court
Newcastle University
Newcastle upon Tyne
NE17RU, UK
rajesh.tiwari@ncl.ac.uk
http://www.ncl.ac.uk
Dr. Samraj Jabez Dhinagar
Vice President, AEG, R\&D
TVS Motor Company Ltd.
PB No.4, Harita
Hosur - 635109
TamilNadu, INDIA
sj.dhinagar@tvsmotor.co.in
http://tvsmotor.in

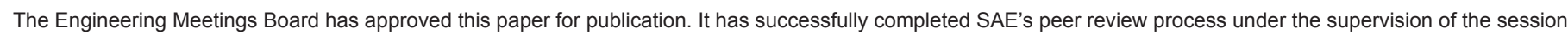
organizer. The process requires a minimum of three (3) reviews by industry experts.

All rights reserved. No part of this publication may be reproduced, stored in a retrieval system, or transmitted, in any form or by any means, electronic, mechanical, photocopying, recording, or otherwise, without the prior written permission of SAE International.

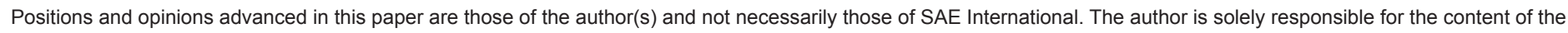
paper.

ISSN 0148-7191

http://papers.sae.org/2014-01-0268 